6 Hopman AHN, Hooren van E, van de Kaa CA, Vooijs GP, Ramaekers FCS. Detection of numerical chromosome aberrations using in situ hybridization in paraffin sections of routinely processed bladder cancers. Mod Pathol of routinely proct

7 Cheung ANY, Sit ASY, Chung LP, Ngan HYS, O'Hanlan $\mathrm{K}$, Wong LC, et al. Detection of heterozygous XY complete hydatidiform mole by chromosome in situ hybridization. Gynecol Oncol 1994;55:386-92.

8 Zanotti F, Mussida M, Merlo D, Meilesi M, Aletti L. Highgrade sarcoma with areas of mullerian adenosarcoma of the uterus. Ann Ostet Ginecol Med Perinat 1991;112:29-35.
9 Chumas JC, Patsner B, Mann WJ. High-grade pelvic sarcoma after radiation therapy for low-grade endometrial stromal sarcoma. Gynecol Oncol 1990;36:428-31.

10 Sabini G, Chumas JC, Mann WJ. Steroid hormone receptors in endometrial stromal sarcomas. A biochemical and immunohistochemical study. Am $f$ Clin Pathol 1992;97:381-6.

11 el Naggar AK, Abdul Karim FW, Silva EG, McLemore D, Garnsey L. Uterine stromal neoplasms: a clinicopathologic and DNA flow cytometric correlation. Hum Pathol 1991;22:897-903.

\title{
Iododeoxyuridine labelling of S-phase fraction in fine needle aspirates from breast carcinomas
}

\author{
R A Maas, P F Bruning, A J Breedijk, J L Peterse
}

\begin{abstract}
The suitability of measuring the S-phase fraction in human breast cancer by labelling tumour cells from fine needle aspirates (FNAs) in vitro with iododeoxyuridine (IdU) was studied in 11 patients. The S-phase fraction was measured both in preoperative FNAs labelled in vitro with IdU, and in FNAs taken from the same tumour when surgically removed after intravenous administration of IdU. Frozen sections were also immunostained for incorporated IdU. The mean S-phase fraction measured in FNAs after in vitro or in vivo labelling and in sections after in vivo labelling was $4.0,3.6$, and 3.1 , respectively. Results of in vitro and in vivo labelling of FNAs with IdU were similar. However, as the S-phase fraction in breast cancer is generally low, the variation between the different measurements is too large; therefore, the S-phase fraction is not a suitable indicator of response to treatment.

$(F$ Clin Pathol 1996;49:607-609)
\end{abstract}

Department of

Pathology,

Netherlands Cancer

Institute/Antoni van

Leeuwenhoekhuis,

Amsterdam,

The Netherlands

R A Maas

A J Breedijk

$\mathrm{J}$ L Peterse

\section{Department of}

Medical Oncology

P F Bruning

Correspondence to: Dr P F Bruning, Netherlands Cancer Institute,

Department of Medical Oncology,

Plesmanlaan 121 1066 CX Amsterdam, The Netherlands.
Keywords: breast cancer, fine needle aspirates, S-phase fraction.

Decreasing proliferative activity in tumours could be used as an early indication of response to systemic treatment. Changes in S-phase fraction as a measure of the proliferative activity could be monitored in sequentially obtained fine needle aspirates (FNAs). Immunostaining of iododeoxyuridine (IdU) incorporated into the DNA in the S-phase of the cell cycle offers a sensitive and specific method to assess $S$-phase fraction in FNAs from breast tumours. In vivo labelling of tumour cells requires the intravenous administration of IdU. Apart from the discomfort to the patient and costs involved, IdU administration may in turn cause further mutation. Moreover, the optimal timing of IdU administration varies from patient to patient. In vitro labelling with IdU is an attractive alternative.

\section{Methods}

IdU LABELLING

The S-phase fraction was measured in 17 patients with primary breast cancer. FNAs were taken from the primary breast carcinomas on the day before surgical removal of the tumour. Viable carcinoma cells were counted using the trypan blue exclusion test. The tumour cells were labelled in vitro by being incubated at $37^{\circ} \mathrm{C}$ for two hours in DMEM culture medium (Gibco BRL, Breda, The Netherlands) containing $10 \%$ fetal calf serum (Gibco BRL) and $10 \mu \mathrm{M}$ IdU (Sigma, Axel, The Netherlands). Approximately six hours before surgery the patients received an intravenous infusion of $100 \mathrm{mg}$ IdU in $50 \mathrm{ml}$. Directly after surgical removal of the tumour a second FNA was taken for the analysis of in vivo labelling. FNAs were washed in phosphate buffered saline (PBS), resuspended in $70 \%$ alcohol and stored at $4^{\circ} \mathrm{C}$ pending analysis. Tumour tissue was snap frozen in liquid nitrogen until further processing.

Permission to administer IdU was obtained from the Medical Ethical Committee of the Antoni van Leeuwenhoekhuis. Informed consent was obtained from each patient.

IMMUNOCYTOCHEMICAL STAINING FOR IdU

Cytospin preparations were prepared, dried and washed in PBS. After being washed in PBS, frozen tumour sections were treated in the same way as the cytospin preparations. All samples were incubated in $95 \%$ formamide in $\mathrm{PBS}$ at $70^{\circ} \mathrm{C}$ for 45 minutes, washed three times for five minutes in $0.1 \mathrm{M}$ Tris $/ \mathrm{HCl}(\mathrm{pH}$ 7.6) supplemented with $5 \%$ Tween 20 , followed by a 10 minute wash in Tris/ $\mathrm{HCl}(\mathrm{pH}$ 7.6). After preincubation for 15 minutes in PBS supplemented with $0.5 \%$ Tween $20,0.1 \%$ bovine serum albumin (BSA) and 10\% normal rabbit serum, the preparations were incubated at room temperature with anti-IdU murine 

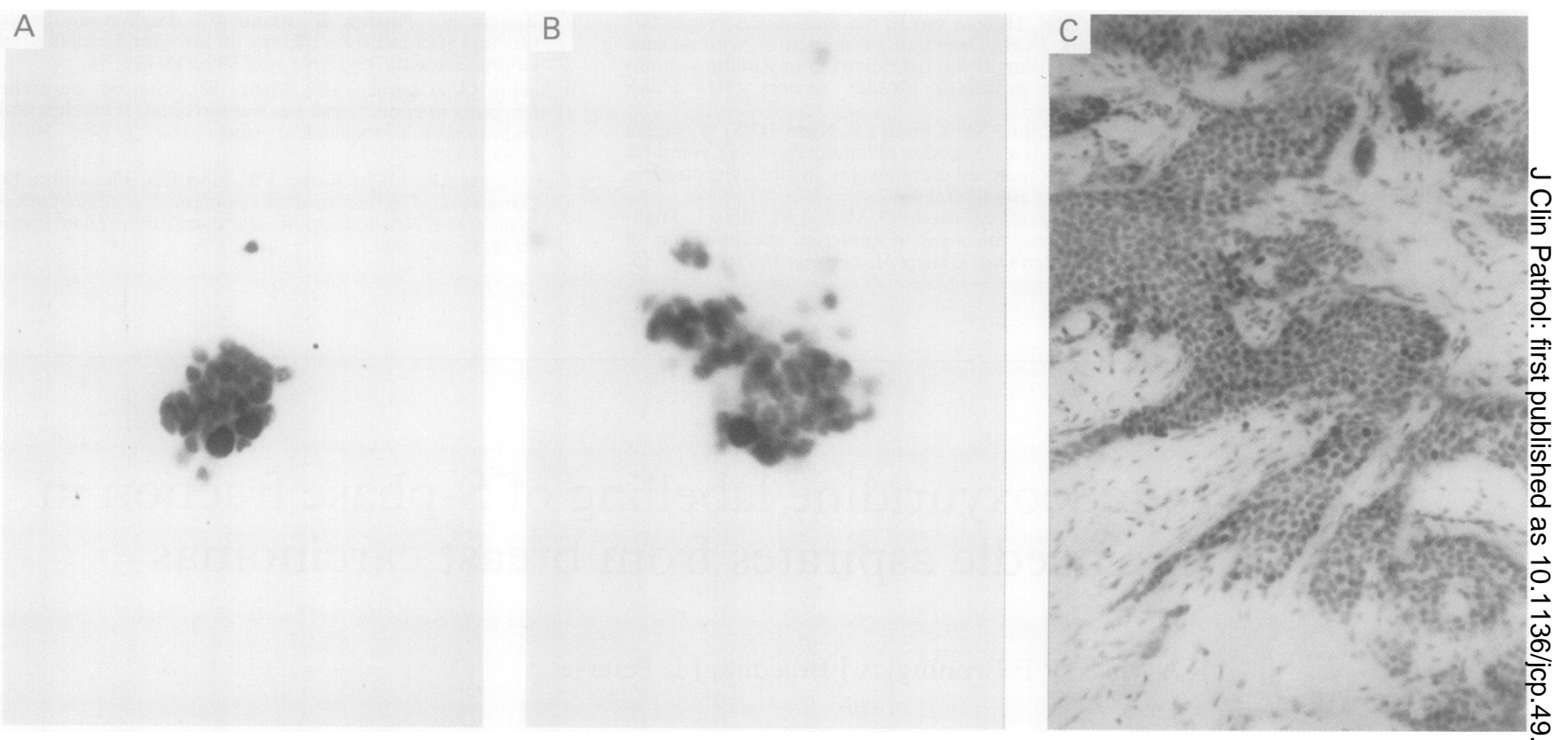

Figure 1. Tumour section and two FNAs from the same patient. (A) FNA after in vitro labelling with IdU; (B) FNA after in vivo labelling with IdU; (C) section after in vivo labelling with IdU.

monoclonal antibody (Becton Dickinson, Etten-Leur, The Netherlands) diluted 1 in 200 in PBS/BSA/Tween/normal rabbit serum. After two washes in PBS/BSA the preparations were incubated in biotinylated rabbit antimouse (Dako, Glostrup, Denmark), diluted 1 in 1000 in $\mathrm{PBS} / \mathrm{BSA} / \mathrm{Tween} /$ normal rabbit serum. Preparations were then washed twice in PBS/BSA followed by a 30 minute incubation with avidin/biotin horse radish peroxidase complex (Dako). After being washed twice in $\mathrm{PBS} / \mathrm{BSA}$ and once in $0.1 \mathrm{M}$ Tris/ $\mathrm{HCl}(\mathrm{pH}$ 7.4), the preparations were incubated with diaminobenzidine/imidazole/hydrogen peroxide in the dark at room temperature for six minutes. Samples were then washed in PBS and staining was enhanced by incubation in copper sulphate $\left(0.5 \% \mathrm{CuSO}_{4} .5 \mathrm{H}_{2} \mathrm{O}, 0.9 \%\right.$ $\mathrm{NaCl}$ ) at room temperature for five minutes. Finally, the preparations were rinsed in aquadest and a general nuclear staining was performed by incubation in haematoxylin for one to three minutes, followed by a four

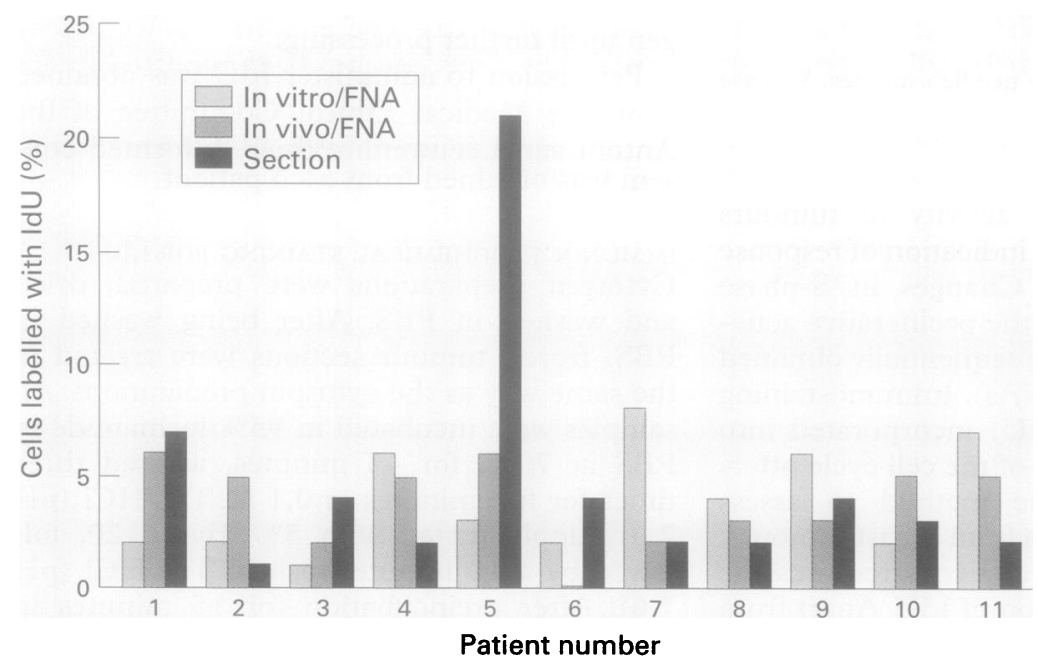

Figure 2 S-phase fraction in 11 patients with breast cancer measured in FNAs after in vitro or in vivo labelling with IdU, or measured on frozen sections after in vivo labelling with IdU.

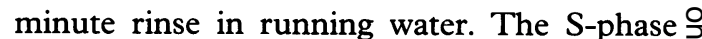
fraction was determined independently by two investigators. At least 1000 tumour nuclei were counted per specimen. Lines were drawn randomly on the glass covering the cell suspen- $\varnothing$ sion and tissue section, and the neighbouring. microscopic fields were analysed.

\section{Results}

The S-phase fraction was determined in 11 of the 17 patients. Too few viable tumour cells $\stackrel{\square}{\varrho}$ were present in one of the two FNAs from the $\overrightarrow{\vec{B}}$ other six. Three different methods were used: 3 (1) in vitro labelling with IdU; (2) in vivo labelling with IdU (FNAs from surgically? resected specimens); and (3) in vivo labelling 웅 with IdU (measured in frozen tumour sections). IdU positive and IdU negative cells 3 . could be distinguished easily in FNA cytospin $\delta$ preparations and the frozen sections (fig 1). Evaluation of S-phase fraction by different 을 observers resulted in excellent conformity, with $\supset$ $<5 \%$ interobserver variation. The data obtained in the 11 patients are summarised in fig $N$ 2 . In one patient a large difference was found between the two FNAs and the frozen section, the latter having a much higher S-phase $\omega$ fraction (3, 6 , and 21 , respectively). This was due to the large number of dead cells present. The mean (SD) S-phase fraction in FNAs in $\mathbb{D}$ the other 10 patients was $4.0(2.5) \%$ after in vitro labelling and $3.6(1.9) \%$ after in vivo 0 labelling; analysis of tissue sections yielded a $\underset{\mathbb{D}}{\mathbb{D}}$ mean S-phase fraction of 3.1 (1.7)\%. The $\overrightarrow{\mathbb{D}}$ mean S-phase fraction values were not signifi- $\frac{2}{0}$ cantly different (paired $t$ test). The mean difference between the measurements on $\frac{0}{0}$ FNAs obtained prior to surgery and those taken from the surgical specimen was $2.6 \%$.

\section{Discussion}

Theoretically, changes in the S-phase fraction, monitored in sequentially obtained FNAs, could act as early indicators of response to therapy, ${ }^{1}$ and can also provide prognostic 
information. ${ }^{2}{ }^{3}$ The incorporation of IdU by tumour cells during S-phase can be measured by immunostaining. This method is more accurate than simple quantification of DNA by FACS analysis and avoids the use of $\left[{ }^{3} \mathrm{H}\right]-$ labelled thymidine. We have studied the reliability of assessing the S-phase fraction in FNAs by in vitro labelling with IdU as a more practical alternative to in vivo labelling. Both methods have disadvantages. In vivo, IdU may not penetrate the tumour completely and intra-tumoral concentrations of IdU vary from patient to patient, adversely affecting the sensitivity of the measurement. In vitro, aspiration may result in cell death, thereby giving a lower S-phase fraction. To control for these potential problems, we also measured the $S$-phase fraction in tumour sections after the in vivo administration of IdU.

The S-phase fraction was measured using all three methods in 11 of the 17 patients included in the study. In one patient the S-phase fraction measured in one of the FNAs was far too low. This was because most cells in the sample were dead. In the other 10 patients, however, no systematic increase or decrease in the S-phase fraction was noted when FNAs and frozen sections were compared. The results obtained with in vitro labelling are generally equivalent to those obtained with in vivo labelling. This suggests that administration of IdU to patients can be avoided.

The S-phase fraction in human breast cancer is generally low, the median value varying between 3.5 and 7.5..$^{3-6}$ Because of this low figure and the variation caused by the labelling methods, analysis of FNAs after in vitro or in vivo labelling with IdU is not suitable for monitoring response to treatment.

1 Fernando IN, Titley JC, Powles TJ, Dowsett M, Trott PA, Ashley SE, et al. Measurement of S-phase fraction and ploidy in sequential fine-needle aspirates from primary human breast tumours treated with tamoxifen. $\mathrm{Br} \mathcal{F}$ Cancer 1994;70:1211-16.

2 Remvikos Y, Beuzeboc P, Zajdela A, Voillemot N, Magdelenat $\mathrm{H}$, Pouillart P. Correlation of pretreatment proliferative activity of breast cancer with the response to cytotoxic chemotherapy. F Natl Cancer Inst 1989;81:1383-7.

3 Gaglia P, Bernardi A, Venesio T, Caldarola B, Lauro D, Cappa APM, et al. Cell proliferation of breast cancer evaluated by anti-brdu and anti-Ki- 67 antibodies: its prognostic value on short-term recurrences. Eur $\mathcal{f}$ Cancer 1993;29A: 1509-13.

4 Rew DA, Campbell ID, Taylor I, Wilson GD. Proliferation indices of invasive breast carcinomas after in vivo 5-bromo-2-deoxyuridine labelling: a flow cytometric study of 75 tumours. Br f Surg 1992;79:335-9.

5 Merlo GR, Venesio T, Bernardi A, Canale L, Gaglia P, Lauro D, et al. Loss of heterozygosity on chromosome 17 p13 in breast carcinomas identifies tumors with high proliferation index. Am F Pathol 1992;140:215-23.

6 Meyer JS, Koehm SL, Hughes JM, Higa E, Wittliff JL, Lagos JA, et al. Bromodeoxyuridine labeling for S-phase measurement in breast carcinoma. Cancer 1993;71:3531-40.

\title{
Sclerosing lymphocytic lobulitis in the male breast
}

\author{
A H S Lee, B Zafrani, G Kafiri, S Rozan, R R Millis
}

\section{Imperial Cancer Research Fund Clinical Oncology Unit, Guy's Hospital, London \\ A H S Lee \\ R R Millis}

\section{Section Medicale et} Hospitaliere, Institut Curie, Paris, France

B Zafrani

S Rozan

\section{Histopathology}

Department,

Hippocration General

Hospital,

Athens, Greece

G Kafir

Correspondence to: Dr A H S Lee, University Department of Pathology,

Mailpoint 813,

Level E, South Block

Southampton Genera

Hospital,

Southampton SO16 6YD.

Accepted for publication 20 February 1996

\begin{abstract}
Sclerosing lymphocytic lobulitis is an inflammatory disorder of the breast that is well recognised in women. It has only been reported previously in two men; two further men with the condition are described here. Both presented with a breast mass, and one was an insulin dependent diabetic. Biopsy specimens from both patients showed circumscribed perivascular and, to a lesser extent, periductal collections of B and T lymphocytes. Sclerosing lymphocytic lobulitis in the female breast shows predominantly perilobular inflammation. The predominantly perivascular distribution in men is consistent with the relative paucity of epithelium in the male breast. Interlobular fibrosis with epithelioid fibroblasts was also present. (f Clin Pathol 1996;49:609-611)
\end{abstract}

Keywords: breast, male, sclerosing lymphocytic lobulitis, inflammation.

Sclerosing lymphocytic lobulitis is a recently recognised disorder of the breast characterised by perilobular and perivascular aggregates of $B$ and $\mathrm{T}$ lymphocytes, with increased expression of class II major histocompatibility antigens by the lobular and ductal epithelium, fibrosis and lobular atrophy. ${ }^{12}$ It is thought to be of autoimmune aetiology and is associated with other autoimmune diseases, particularly diabetes mellitus. The condition is well recognised in women, but has been reported in only two men. ${ }^{3}$ We describe two additional male patients in whom the perivascular and periductal inflammation has been characterised immunohistochemically.

\section{Case reports}

Patient 1 was a 47 year old Algerian man who presented with a $15 \mathrm{~mm}$ mass in the right breast. He had had insulin dependent diabetes mellitus for 31 years, but no history of other autoimmune diseases. Patient 2 was a 53 year old Greek man who presented with a poorly defined $5 \mathrm{~cm}$ breast mass. He did not have diabetes mellitus nor any other autoimmune disease, nor a family history of diabetes.

PATHOLOGICAL FINDINGS

An excision biopsy specimen was taken of the breast mass of both men. Sections from both 